Claremont Colleges

Scholarship@ Claremont

All HMC Faculty Publications and Research

HMC Faculty Scholarship

$12-1-1993$

\title{
Short Terahertz Pulses from Semiconductor Surfaces: The Importance of Bulk Difference-Frequency Mixing
}

Peter N. Saeta

Harvey Mudd College

Benjamin I. Greene

AT\&T Bell Laboratories

Shun Lien Chuang

University of Illinois at Urbana-Champaign

\section{Recommended Citation}

"Short terahertz pulses from semiconductor surfaces: The importance of bulk difference-frequency mixing," P. N. Saeta, B. I. Greene, and S. L. Chuang, Appl. Phys. Lett. 63, 3482 (1993). doi: 10.1063/1.110127

This Article is brought to you for free and open access by the HMC Faculty Scholarship at Scholarship @ Claremont. It has been accepted for inclusion in All HMC Faculty Publications and Research by an authorized administrator of Scholarship @ Claremont. For more information, please contact scholarship@cuc.claremont.edu. 


\section{AIP Appiled Physics \\ Letters}

\section{Short terahertz pulses from semiconductor surfaces: The importance of bulk differencefrequency mixing}

Peter N. Saeta, Benjamin I. Greene, and Shun Lien Chuang

Citation: Appl. Phys. Lett. 63, 3482 (1993); doi: 10.1063/1.110127

View online: http://dx.doi.org/10.1063/1.110127

View Table of Contents: http://apl.aip.org/resource/1/APPLAB/v63/i25

Published by the AIP Publishing LLC.

Additional information on Appl. Phys. Lett.

Journal Homepage: http://apl.aip.org/

Journal Information: http://apl.aip.org/about/about_the_journal

Top downloads: http://apl.aip.org/features/most_downloaded

Information for Authors: http://apl.aip.org/authors

\section{ADVERTISEMENT}
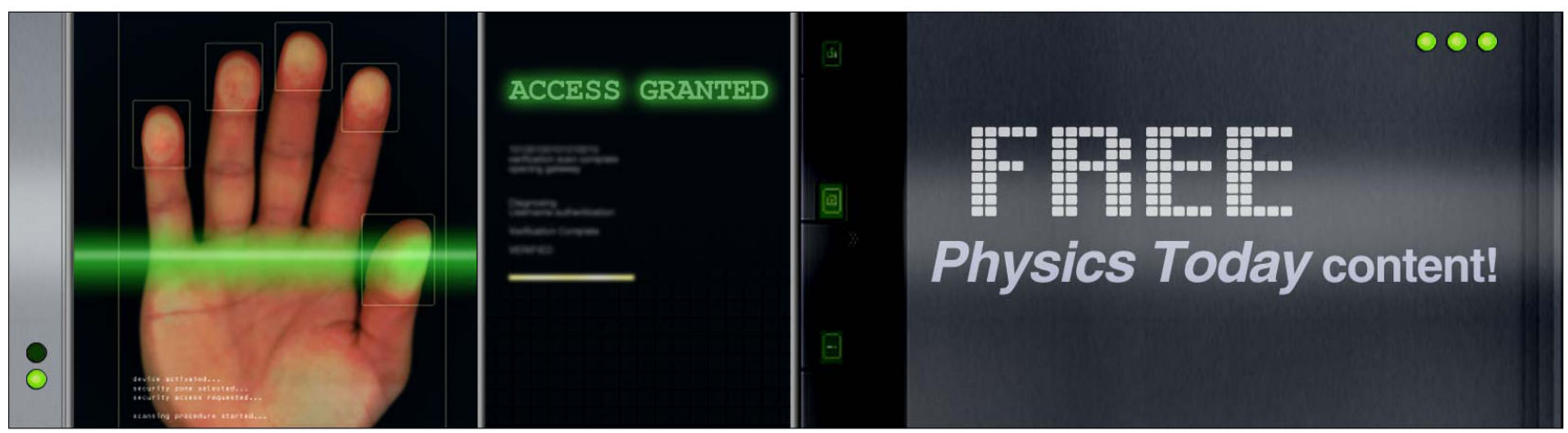


\title{
Short terahertz pulses from semiconductor surfaces: The importance of bulk difference-frequency mixing
}

\author{
Peter N. Saeta ${ }^{a)}$ and Benjamin I. Greene \\ AT\&T Bell Laboratories, 600 Mountain Avenue, Murray Hill, New Jersey 07974 \\ Shun Lien Chuang \\ Department of Electrical and Computer Engineering, University of Illinois at Urbana-Champaign, \\ Urbana, Illinois 61801
}

(Received 18 June 1993; accepted for publication 11 October 1993)

\begin{abstract}
The crystallographic orientation dependence of the far-infrared (FIR) light generated at the (001) surface of a zincblende semiconductor is shown to derive principally from bulk difference-frequency mixing. A strong modulation is observed for $1-\mathrm{GW} / \mathrm{cm}^{2}$ pulses on InP, which demonstrates that the radiated FIR wave produced by bulk optical rectification is comparable to that generated by the transport of photoinjected carriers. Using the bulk rectification light as a clock, we show that more than $95 \%$ of the light produced from an InP (111) crystal by $100-\mathrm{fs}, 100-\mu \mathrm{J}$ pulses is generated in a time shorter than the excitation pulse.
\end{abstract}

A subpicosecond pulse of visible light reflected of the surface of a semiconductor generates a short pulse of farinfrared (FIR) light in the direction of specular reflection. ${ }^{1,2}$ This phenomenon was first explained as the dipole radiation of a time-changing current of photoinjected charge carriers in the static depletion field near the semiconductor surface. This current surge mechanism successfully explained the observed intensity maximum for incidence near the Brewster angle and the TM polarization of the emitted FIR radiation. However, it was unsuccessful in accounting for the modulation of the FIR light observed at high intensity as the crystalline semiconductor wafer was rotated about the surface normal. An alternative explanation was proposed ${ }^{3}$ in which the FIR light was produced by difference-frequency mixing from an effective secondorder nonlinear susceptibility proportional to the depletion field. A calculation of the strength of this field-induced optical rectification mechanism suggested that it could account for the observed magnitude of FIR light and its dependence on polarization and polarity of the surface field. It was furthermore suggested ${ }^{3}$ that the crystallographic dependence of the FIR intensity could be simply explained by this nonlinear optical model. Field-induced optical rectification has recently been observed from biased quantum wells, in which the perpendicular transport of charges was suppressed by the barriers. ${ }^{4} \mathrm{~A}$ third mechanism, difference-frequency mixing from the bulk secondorder susceptibility, or bulk optical rectification, was assumed to be negligible.

In this letter we assess the relative strength of these three mechanisms in InP, the semiconductor observed to produce the most FIR light. Our principal findings are (a) of the three generation mechanisms, only bulk rectification causes modulation with rotation on a (001) surface, and bulk rectification causes the preponderance of rotation dependence on a (111) surface; (b) the relative importance

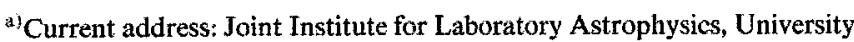
of Colorado, Boulder, CO 80309-0440.
}

of bulk rectification grows dramatically with incident visible intensity, becoming comparable in magnitude to the field-dependent mechanisms at intensities of order $1 \mathrm{GW} /$ $\mathrm{cm}^{2}$; and (c) on a (111) surface over $95 \%$ of the FIR light energy is generated in a time shorter than the excitation pulse width. The relative rise in importance of bulk rectification is most likely caused by reduction of the depletion field by the photoinjected carriers.

The ability to discriminate between the three mechanisms comes from their different crystallographic and temporal dependence. We used the following experimental geometry. The collimated excitation beam $(100 \mathrm{fs}, 2.0 \mathrm{eV}$, $630 \mathrm{~nm}$ ) was polarized in the plane of incidence (TM) and incident on the surface of the InP crystal at a fixed angle $\theta_{i}$. The crystal was mounted on a computer-controlled stage that allowed it to be rotated through an angle $\phi$ about the surface normal. The reflected FIR pulse then passed through a free-standing wire-grid polarizer oriented to transmit either the TE or the TM light, which was detected by a He-cooled bolometer.

FIR light generated by the current-surge mechanism does not depend on crystallographic orientation because the linear dielectric properties of cubic crystals are isotropic, and because the mass tensor for electrons in the $\Gamma$ valley of InP and GaAs is nearly isotropic. This light gives a $\phi$-independent contribution to the measured signal. Recently it has been pointed out that for very short times, before photoinjected carriers can scatter off phonons, the ballistically accelerated carriers are sensitive to the crystal symmetry. ${ }^{5}$ Nonetheless, little crystallographic dependence was observed at low optical intensity, ${ }^{6}$ which demonstrates that the vast majority of the FIR light produced by the current-surge mechanism is insensitive to crystal orientation. We therefore make the working definition that the current-surge light is independent of azimuthal angle.

FIR light generated by bulk optical rectification is governed by the symmetry of the second-order susceptibility tensor, $\chi^{(2)}$, and is given by the expression 


$$
\begin{aligned}
E_{i}^{\mathrm{fIR}}(\Omega) & \propto \frac{\partial^{2} p_{i}(\Omega)}{\partial t^{2}} \\
& \equiv \frac{\partial^{2}}{\partial t^{2}} \chi_{i j k}^{\partial t^{2}}\left(\Omega=\omega_{1}=\omega_{2} ; \omega_{1},-\omega_{2}\right) E_{j}\left(\omega_{1}\right) E_{k}^{*}\left(\omega_{2}\right),
\end{aligned}
$$

where $\omega_{1}$ and $\omega_{2}$ are optical frequencies, $E$ is the electric field in the nonlinear medium, $P$ is the induced nonlinear polarization at the difference frequency $\Omega=\omega_{1}-\omega_{2}$, and repeated indices are summed over. In zincblende crystals, such as InP and GaAs, the only nonvanishing components of $\chi_{i j k}^{(2)}$ in the cartesian frame of the conventional unit cell are those without a repeated index, such as $\chi_{x y z}^{(2)}{ }^{7}$ From this one can show that for fixed $\theta_{i}$ on a (001) surface the generated TM field component is proportional to $\sin 2 \phi$, and the TE field component is proportional to $\cos 2 \phi$, where $\phi$ is measured from the [100] axis. On a (111) surface, the TE wave is proportional to $\sin 3 \phi$, where $\phi$ is measured from the [211] direction, and the TM wave has a component proportional to $\cos 3 \phi$ and (away from normal incidence) one independent of $\phi$.

The FIR polarization produced by field-induced optical rectification is given by the expression ${ }^{3}$

$$
\begin{aligned}
P_{i}(\Omega) & =\chi_{i j k l}^{(3)}(\Omega \\
& \left.=\omega_{l}-\omega_{2} ; \omega_{1},-\omega_{2}, 0\right) E_{j}\left(\omega_{1}\right) E_{k}^{*}\left(\omega_{2}\right) E_{l}(0),
\end{aligned}
$$

where $E(0)$ is the static depletion field directed along the surface normal. In zincblende crystals the nonvanishing components of $x_{i j k l}^{(3)}$ are $x x x x=y y y y=z z z z, x x y y=y y x x$ $=y y z z=z z y y=x x z z=z z x x, \quad x y x y=y x y x=x z x z=z x z x$ $=y z y z=z y z y$, and $x y y x=y x x y=x z z x=z x x z=y z z y=z y y z,^{7}$ but due to frequency degeneracies $\left(\omega_{1} \approx \omega_{2}\right.$ and $\left.\Omega \approx 0\right)$, the three independent components are $x x x x, x y y x$, and $x y x y$ $=x x y y$. On a (001) surface these components give rise to a TM-polarized FIR wave that has no dependence on the rotation angle $\phi$, regardless of the polarization of the input. 'Thus any TE-polarized FIR light must come from the bulk $x^{(2)}$, and any sinusoidal modulation of the TM-polarized FIR light with $\phi$ must also come from bulk rectification. On a (111) surface the $\phi$-dependent contribution to the TM (TE) wave is proportional to $\Gamma \cos 3 \phi(\Gamma \sin 3 \phi)$, where $\Gamma \equiv-\chi_{x x x x}+\chi_{x z z x}+2 \chi_{x z x z}$. Zhang et al. have recently observed that the polarity of the radiated wave generated at a GaAs (111) surface at normal incidence does not reverse when the direction of a $11-\mathrm{kV} / \mathrm{cm}$ static field is reversed, from which we infer that $\left|\Gamma E_{\mathrm{dc}}<\right| \chi_{x y z}^{(2)}$. As for a (001) surface, the modulation for a (111) surface is therefore principally due to bulk rectification. There is also a $\phi$-independent contribution to the TM FIR wave, which includes a normally directed polarization proportional to $I I \equiv \chi_{x x x x}-2 \chi_{x z x z}+2 \chi_{x z z x}$.

Figure 1 shows the measured TE- and TM-polarized FIR intensity generated from an InP (001) surface as a function of rotation angle $\phi$. The excitation fluence and $\theta_{i}$ were $120 \mu \mathrm{J} / \mathrm{cm}^{2}$ and $66^{\circ}$ for the TM data and $250 \mu \mathrm{J} / \mathrm{cm}^{2}$ and $45^{\circ}$ for the TE data, which have been magnified by a factor of 5 for clarity. From the foregoing discussion we know that the TE light arises solely from bulk rectification

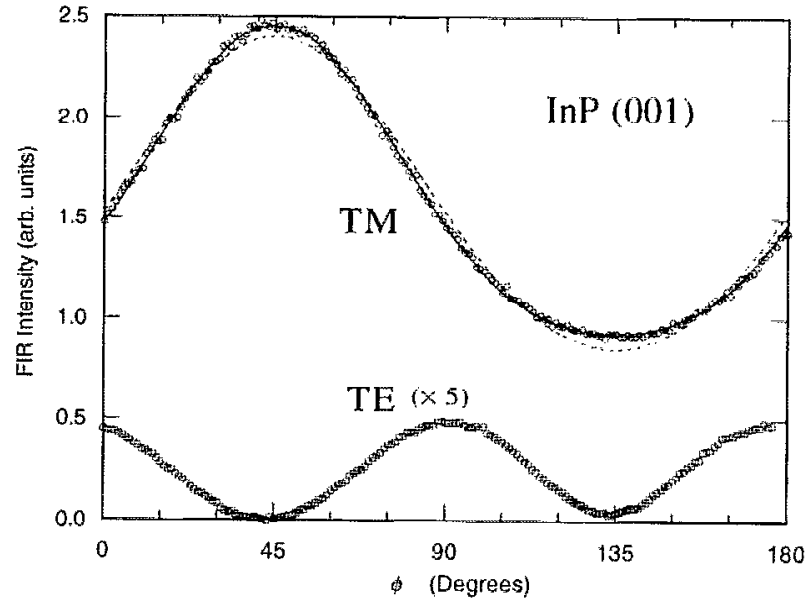

FIG. 1. TM- and TE-polarized FIR intensity for 100-fs, 2-eV, TM excitation pulses incident on an InP (001) wafer as a function of rotation angle $\phi$ measured from the [100] axis. The incidence angle was $66^{\circ}$ for the TM data, and $45^{\circ}$ for the TE data, which have been magnified by a factor of 5 for clarity. Curves through the TM data are fits to $I^{\mathrm{FIR}}(\phi)=\left(c_{1}\right.$ $\left.+c_{2} \sin 2 \phi\right)^{2}+c_{3}^{2}$; for the broken line, $c_{3}=0$. The solid line gives $c_{1}+c_{2}+c_{3}$ $=1: 0.58: 1.11$.

and its amplitude is proportional to $\cos 2 \phi$. Since the bolometer detects the integrated intensity, the observed signal is proportional to $\cos ^{2} 2 \phi$.

All three mechanisms contribute to the TM light and we expect the FIR intensity to be $I^{\mathrm{FIR}} \propto\left(E^{\mathrm{FIR}}\right)^{2}=\left(c_{1}\right.$ $\left.+c_{2} \sin 2 \phi\right)^{2}$, where $c_{1}$ is the sum of contributions from the current-surge and field-induced rectification and $c_{2}$ expresses the contribution from bulk rectification. The best fit to this function is shown by the dashed line in the figure; although the correlation is reasonably good, a much better fit is obtained using the more general function

$$
I^{\mathrm{FIR}}=\left(c_{1}+c_{2} \sin 2 \phi\right)^{2}+c_{3}^{2} .
$$

The coefficient $c_{3}$ here represents a $\phi$-independent contribution to the detected light that does not interfere with the bulk-rectification term. This term arises from the different duration of the current-surge pulse. Both the bulkrectification and field-induced optical rectification mechanisms are instantaneous, whereas the acceleration of photoinjected carriers is expected to continue for some time after the excitation pulse. The tail of the current-surge pulse, therefore, has no bulk-rectification light with which to interfere, and so appears as a constant offset. The fit gives the coefficients $c_{i}$ in the ratios $c_{1}: c_{2}: c_{3}-1.72: 1: 1.92$, which shows that more than half the FIR light is generated by the instantaneous mechanisms at this high excitation intensity.

Greater modulation with $\phi$ was observed on an InP (111) surface. In Fig. 2 the normalized TM-polarized FIR intensity is plotted versus $\phi$ for three angles of incidence. The excitation fluence was greater than $140 \mu \mathrm{J} / \mathrm{cm}^{2}$ for each trace. The data were fitted to the function

$$
I^{\mathrm{FIR}}(\phi)=\left(c_{1}+c_{2} \cos 3 \phi\right)^{2}+c_{3}^{2},
$$

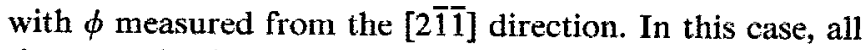
three mechanisms contribute to $c_{1}, c_{2}$ comes from bulk 


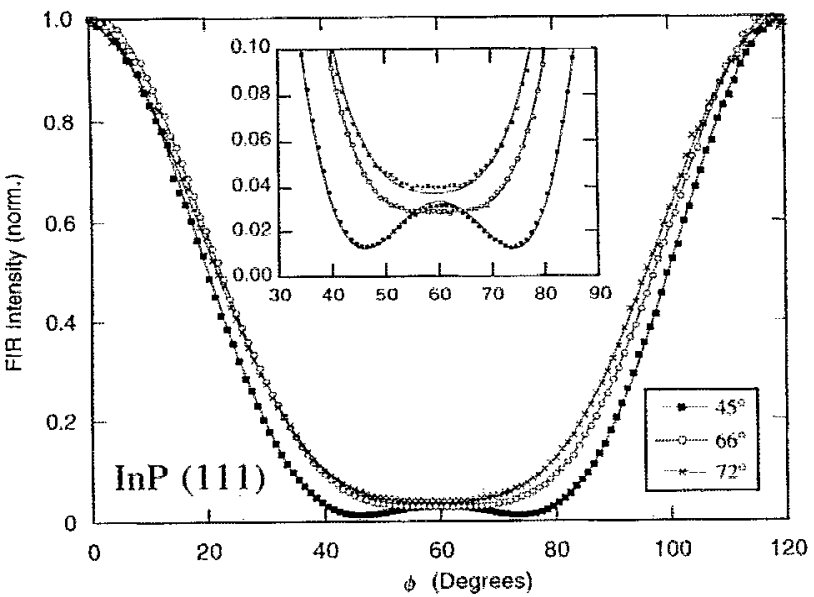

FIG. 2. Normalized TM-polarized FIR intensity from an $\operatorname{InP}$ (111) surface vs rotation angle $\phi$ for incidence angles $\theta_{i}=45^{\circ}, 66^{\circ}$, and $72^{\circ}$. The TM-polarized excitation fluence was greater than $140 \mu \mathrm{J} / \mathrm{cm}^{2}$ for each curve. Curves through the data points are fits to $I^{\mathrm{FIR}}(\phi)=\left(c_{1}\right.$ $\left.+c_{2} \cos 3 \phi\right)^{2}+c_{3}^{2}$. The inset shows data and fits near the minimum on an expanded vertical scale.

rectification, and the tail of the current-surge light gives rise to $c_{3}$ as before. The fits are excellent and show that almost all the energy $(>95 \%)$ is contained in the instantaneous terms $c_{1}$ and $c_{2}$; there is relatively very little current-surge tail. This is primarily due to the more efficient coupling of the optical fields to the symmetry-allowed components of $\chi^{(2)}$ on a (111) surface. Furthermore, the data show increasing modulation as $\theta_{i}$ decreases, with a zero crossing near $\phi=46^{\circ}$ for $\theta_{i}=45^{\circ}$. This dependence can be understood qualitatively by noting that the normally directed current or polarization radiates with an efficiency proportional to $\theta_{i}$ for small $\theta_{i}$, vanishing at normal incidence. ${ }^{1}$ Bulk rectification, however, has a transverse component that radiates with decreasing efficiency away from normal incidence. The combination of these two effects thus leads to decreasing modulation with $\theta_{i}$. Since the instantaneous terms produce a rectified wave according to $E^{\mathrm{FIR}}(t) \propto \partial^{2} P^{(\mathrm{NL} . \mathrm{j}} / \partial t^{2} \propto \partial^{2} I_{\text {vis }} / \partial t^{2}$, the pulse duration of the FIR wave is even shorter than that of the optical excitation pulse. ${ }^{9}$

In summary, we have analyzed the FIR pulse generated from InP (001) and (111) surfaces by high-intensity $\sim 100$ - $f$ s above-band-gap pulses to determine the relative contribution of the current surge, bulk rectification, and field-induced optical rectification mechanisms. The strong modulation of the generated FIR intensity as the sample is rotated about the surface normal demonstrates the increasing importance of the bulk-rectification contribution as the excitation intensity is increased above $\sim 10 \mu \mathrm{J} / \mathrm{cm}^{2}$. This relative growth arises because bulk rectification does not require the surface field, as the other two mechanisms do, and is thus less susceptible to screening. Above $\sim 100 \mu \mathrm{J} /$ $\mathrm{cm}^{2}$ the degree of modulation remains nearly constant with intensity, showing that all significant mechanisms saturate equally at high intensity. A simple Drude calculation of the attenuation length of the FIR wave caused by the photoexcited plasma, based on the measured terahertz mobility ${ }^{10}$ gives a value only slightly longer than the penetration depth of the excitation pulse, indicating appreciable absorption of the gencrated FIR. In this high-fluence regime, optical rectification was observed to account for $\sim 65 \%$ of the light on a (001) surface, and up to $>95 \%$ on a (111) surface. A constant base line offset in the rotational dependence data demonstrates the presence of a long-lived tail to the light generated by the photoinjected current for nonnormal incidence.

The strength of bulk rectification at high excitation intensity implies that very broadband $\mathrm{THz}$ pulses can be generated from InP surfaces in a geometry that excludes radiation from transport; i.e., along the normal of a (111) surface. The duration of such pulses will be determined by that of the visible excitation pulse, except when the bandwidth of the (transform-limited) excitation pulse approaches the rehstrahlen peak at $9.2 \mathrm{THz}$, which may cause ringing and spectral narrowing. Furthermore, difference-frequency mixing from two distinct femtosecond pulses should provide a simple and reasonably efficient means of generating short pulses of tunable light in the FIR and IR.

S. L. C. thanks C. S. Chang for helpful discussions and the Office of Naval Research for support under Grant No. N00014-90-J-1821.

'X.-C. Zhang, B. B. Hu, J. T. Darrow, and D. H. Auston, Appl. Phys. Lett. 56, 1011 (1990).

${ }^{2}$ X.-C. Zhang, J. T. Darrow, B. B. Hu, D. H. Auston, M. T. Schmidt, P, Tham, and E. S. Yang, Appl. Phys. Lett. 56, 2228 (1990).

${ }^{3}$ S. L. Chuang, S. Schmitt-Rink, B. I. Greene, P. N. Saeta, and A. F. J. Levi, Phys. Rev. Lett. 68, 102 (1992).

${ }^{4}$ P. C. M. Planken, M. C. Nuss, W. H. Knox, D. A. B. Miller, and K. W. Goossen, Appl. Phys. Lett. 61, 2009 (1992).

${ }^{5}$ A. V. Kuznetsor and C. J. Stanton, Bull. Am. Phys. Soc. 38, 687 (1993).

${ }^{6}$ X.-C. Zhang and D. H. Auston, J. Appl. Phys. 71, 326 (1992).

${ }^{7}$ Y. R. Shen, The Principles of Nonlinear Optics (Wiley, New York, 1984).

${ }^{8}$ X.-C. Zhang, Y. Jin, L. Kingsley, and M. Weiner, Appl. Phys. Lett. 62, 2477 (1993).

${ }^{9}$ It should be noted that if the ratio $c_{1} / c_{2}$ were complex (and equal to $a+i b)$, the FIR intensity would be given by $I^{\mathrm{FlR}}(\phi)=\left|c_{2}\right|{ }^{2}[(a$ $\left.+\cos 3 \phi)^{2}+b^{2}\right]$, which has the same form as Eq. (4). There is the mathematical possibility, therefore, that the base line offset can be attributed to a phase difference between the current-surge and opticalrectification radiation. However, the small offset evident in the (111) data for $\theta_{i}=45^{\circ}$ puts an upper limit of 0.2 on $|b / a|$, showing that the in-phase term dominates. Furthermore, one expects from the transport properties of a high purity crystalline semiconductor that the acceleration of injected charges will persist beyond the 100-fs optical pulse, making the interpretation given in the text based on temporal delay much more likely.

${ }^{10}$ P. N. Saeta, J. F. Federici, B. I. Greene, and D. R. Dykaar, Appl. Phys. Lett. 60, 1477 (1992). 